\title{
Transient Analysis and Design Improvement of a Gas Turbine Rotor Based on Thermal-Mechanical Method
}

\author{
Yang Liu $\mathbb{D}^{1,2}$ Qi Yuan $\mathbb{D}^{1,2}$ Guangyu $\mathrm{Zhu},{ }^{1,2}$ and $\mathrm{Pu} \mathrm{Li}^{1,2}$ \\ ${ }^{1}$ School of Energy and Power Engineering, Xian Jiaotong University, Xi'an 710049, China \\ ${ }^{2}$ Shaanxi Engineering Laboratory of Turbomachinery and Power Equipment, Xian 710049, China
}

Correspondence should be addressed to Qi Yuan; qyuan@xjtu.edu.cn

Received 11 January 2018; Revised 21 March 2018; Accepted 1 April 2018; Published 8 May 2018

Academic Editor: Mickaël Lallart

Copyright (c) 2018 Yang Liu et al. This is an open access article distributed under the Creative Commons Attribution License, which permits unrestricted use, distribution, and reproduction in any medium, provided the original work is properly cited.

\begin{abstract}
The rotor is the core component of a gas turbine, and more than $80 \%$ of the failures in gas turbines occur in the rotor system, especially during the start-up period. Therefore, the safety assessment of the rotor during the start-up period is essential for the design of the gas turbine. In this paper, the transient equivalent stress of a gas turbine rotor under the cold start-up condition is investigated and the novel tie rod structure is introduced to reduce the equivalent stress. Firstly, a three-dimensional finite element model of the gas turbine rotor is built, and nonlinear contact behaviors such as friction are taken into account. Secondly, the convective heat transfer coefficients of the gas turbine rotor under the cold start-up condition are calculated using thermal dynamic theory. The transient analysis of the gas turbine rotor is conducted considering the thermal load, the centrifugal load, and the pretightening force. The temperature and stress distributions of the rotor under the cold start-up condition are shown in detail. In particular, the generation mechanism of maximum equivalent stress for tie rods and the change tendency of the pretightening force are illustrated in detail. The tie rod holes of the rear shaft and the turbine tie rod are the dangerous locations during the startup period. Finally, a novel tie rod is proposed to reduce the maximum equivalent stress at the dangerous location. The maximum equivalent stress at this location is decreased by $15 \%$. This paper provides some reference for the design of the gas turbine rotor.
\end{abstract}

\section{Introduction}

The gas turbine rotor is operating under the condition of high temperature, high pressure, and high rotational speed. The complex loads have significant impact on the rotor. In order to identify the temperature and stress distributions of the rotor, developing an accurate model for the temperature and stress analysis is very important. There have been many researches on the temperature and stress fields of the gas turbine rotor. By applying the finite element method, the maximum stress of a gas turbine rotor was discussed considering pretightening force, centrifugal force, and torque [1]. The effects of static installation clearances, shoulder numbers, and spans on the stress of the tie rod were investigated [2]. The temperature and thermal stress distribution of a gas turbine rotor were analyzed under different transient conditions based on a two-dimensional finite element model, which provided reference for the stress analysis of gas turbine rotor [3]. A thermal-mechanical coupled method was developed to calculate the thermal stress field of a steam turbine rotor, which was more accurate compared with the uncoupled method [4]. The steady and transient analysis of two real gas turbine rotors was presented under various loads using three-dimensional finite model [5]. The high stress areas were predicted in two F-class gas turbine rotors under steadystate and transient conditions [6]. The stress distribution and contact status of curvic couplings were studied in a gas turbine rotor with consideration of pretightening force, centrifugal force, torque, and steady-state thermal load [7]. There are also some researches about the contact analysis. The connection effect at nominal interference of the turbine rotor was analyzed [8]. The contact modelling of the interference fit was established for fretting fatigue strength calculations using finite analysis [9].

Besides, the prediction of the turbine rotor failure location has been conducted in some papers. Based on the finite element method, the failure location and life consumption of a gas turbine disc were predicted, and the results were 


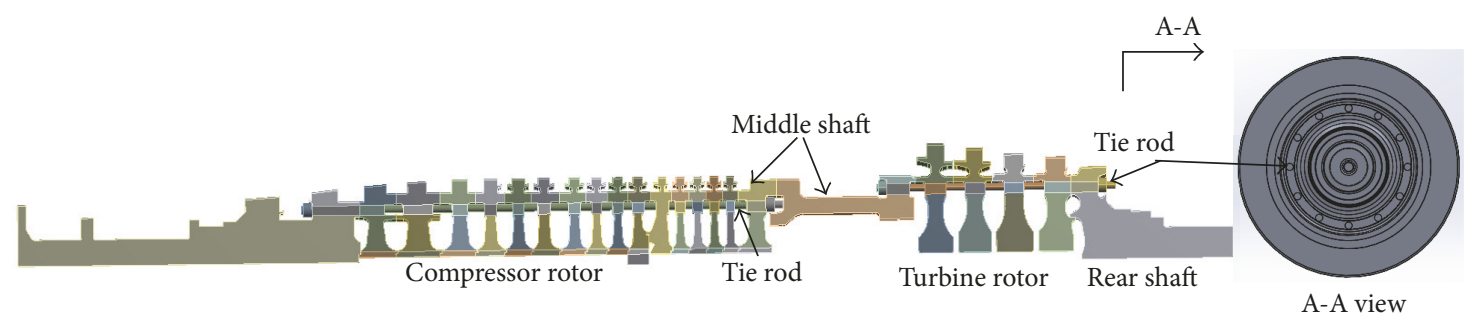

FIGURE 1: The structure of the gas turbine rotor.

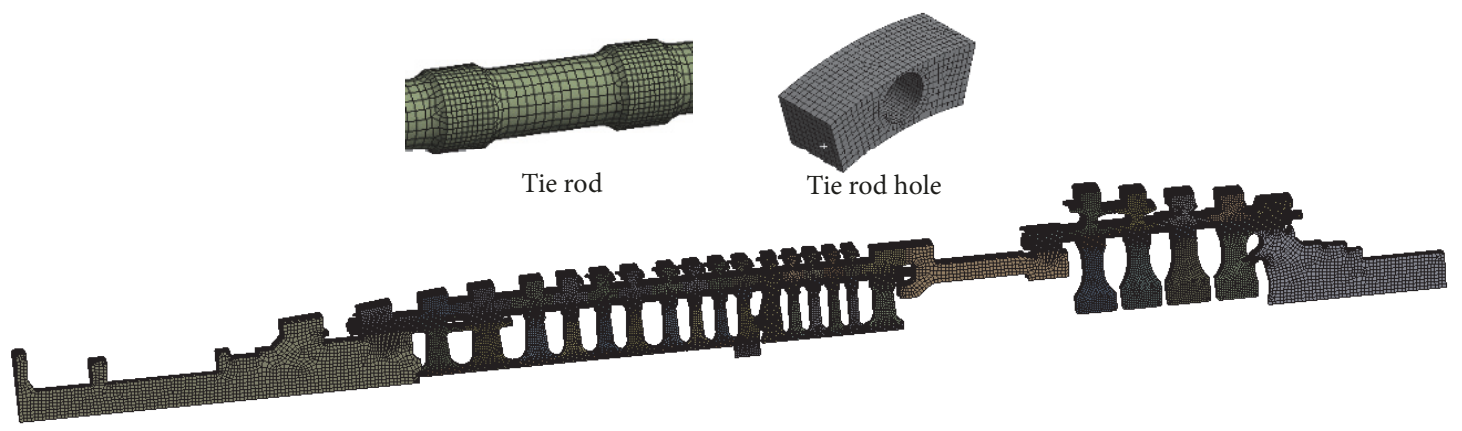

FIgURE 2: A one-twelfth cyclic symmetry three-dimensional finite element model of the gas turbine rotor.

also compared with experimental data obtained from the real model disc [10]. Several approaches were adopted to investigate the failure of a $60 \mathrm{MW}$ turbine rotor. The results showed that the fatigue mechanism derived from the stress field generated by the frequent start-up and the blade fixing method [11]. Based on the thermal stress analysis of a turbine rotor at transient condition, the fatigue life of the rotor was obtained by solving the Green function [12]. A nonlinear damage model was established to analyze the fatigue damage of a rod-fastened rotor under the start-up condition [13]. Manson-Coffin equation was used to predict the failure location of a rod-fastened rotor based on a two-dimensional finite element model at transient condition [14]. Because the great temperature gradient and thermal stress will diminish the fatigue life of the gas turbine rotor, high-performance materials were introduced to ensure the operating security [15-19]. Apart from the material selection, the optimization method was necessary for minimizing the stress and the fatigue consumption of the turbine rotor. The structure and operation optimization of a supercritical steam turbine rotor were conducted, and the fatigue life of the rotor was improved by optimizing the start-up curve and the shape of selected areas [20].

However, there are few investigations that focus on the stress distributions of the gas turbine rotor with tie rods and holes considering all kinds of loads during cold start-up period. Since tie rods and holes are distributed in the circumferential direction, the variation of tightening force of the tie rod or the interaction between tie rod shoulders and holes should be evaluated. Meanwhile, there is no research focusing on the design improvement of the tie rod structure. In this paper, a three-dimensional finite element model of the gas turbine rotor is built, and nonlinear contact behaviors such as friction are taken into account. The transient analysis of the gas turbine rotor is conducted considering thermal load, centrifugal load, and pretightening force. The temperature and stress distributions of the rotor under the cold start-up condition are shown in detail. Finally, a novel tie rod structure is introduced to reduce the maximum equivalent stress at the dangerous location.

\section{Modelling of the Gas Turbine Rotor}

2.1. Introduction of the Gas Turbine Rotor. The investigated gas turbine rotor is obtained from cooperation with a turbine company. Figure 1 shows the schematic diagram of the gas turbine rotor. The rotor consists of 17-stage compressor rotor, 4-stage turbine rotor, two middle shafts, and a rear shaft. The compressor rotor and turbine rotor are held together by 12 tie rods, respectively. The compressor rotor is connected with the turbine rotor through two middle shafts. Considering the structure of tie rods, the three-dimensional model is applied in the analysis.

2.2. Modelling and Material Properties. Owing to the cyclic symmetry structure of the rotor (the number of tie rods is 12), a one-twelfth cyclic symmetry model (with one tie rod) is built. The cycle symmetry three-dimensional finite element model of the rotor is shown in Figure 2. This model involves five kinds of element types, including solid element SOLID186, thermal element SOLID90, contact element CONTA174, TARGE170, and preload element PRETS179 [21]. This model also includes three kinds of contact interfaces, namely, contact interfaces between two discs, nuts of tie rods, and discs, tie rods, and the sidewalls of tie rod holes. The contact type of all the contact surfaces is frictional 
TABLE 1: Material properties of the gas turbine rotor (compressor discs).

\begin{tabular}{lccccccc}
\hline Temperature & ${ }^{\circ} \mathrm{C}$ & 20 & 100 & 200 & 300 & 400 & 500 \\
\hline Thermal conductivity & $\mathrm{W} \cdot(\mathrm{m} \cdot \mathrm{K})^{-1}$ & 34.6 & $/$ & 39.6 & 38.2 & 36.4 & 34.4 \\
Young's modulus & $\mathrm{GPa}$ & 204 & 201 & 196 & 190 & 182 & 173 \\
Coefficients of thermal expansion & $10^{-6}{ }^{\circ} \mathrm{C}^{-1}$ & 11.7 & 11.7 & 12.3 & 12.7 & 13.1 & 13.3 \\
Poisson's ratio & $/$ & & & & 0.31 & & \\
Density & $\mathrm{Kg} \cdot \mathrm{m}^{-3}$ & & & & 7860 & \\
\hline
\end{tabular}

TABLE 2: Material properties of the gas turbine rotor (tie rods and turbine discs).

\begin{tabular}{lcccccccc}
\hline Temperature & ${ }^{\circ} \mathrm{C}$ & 20 & 100 & 200 & 300 & 400 & 500 & 600 \\
\hline Thermal conductivity & $\mathrm{W} \cdot(\mathrm{m} \cdot \mathrm{K})^{-1}$ & $/$ & 13.1 & 15.4 & 17.3 & 18.9 & 20.2 & 21.0 \\
Young's modulus & $\mathrm{GPa}$ & 208 & 207 & 202 & 196 & 189 & 182 & 175 \\
Coefficients of thermal expansion & $10^{-6}{ }^{\circ} \mathrm{C}^{-1}$ & $/$ & 13.0 & 13.3 & 13.8 & 14.3 & 15.0 & 15.6 \\
Poisson's ratio & $/$ & 0.32 & 0.33 & 0.32 & 0.33 & 0.34 & 0.35 & 0.36 \\
Density & $\mathrm{Kg} \cdot \mathrm{m}^{-3}$ & & & & 8259 & & \\
\hline
\end{tabular}

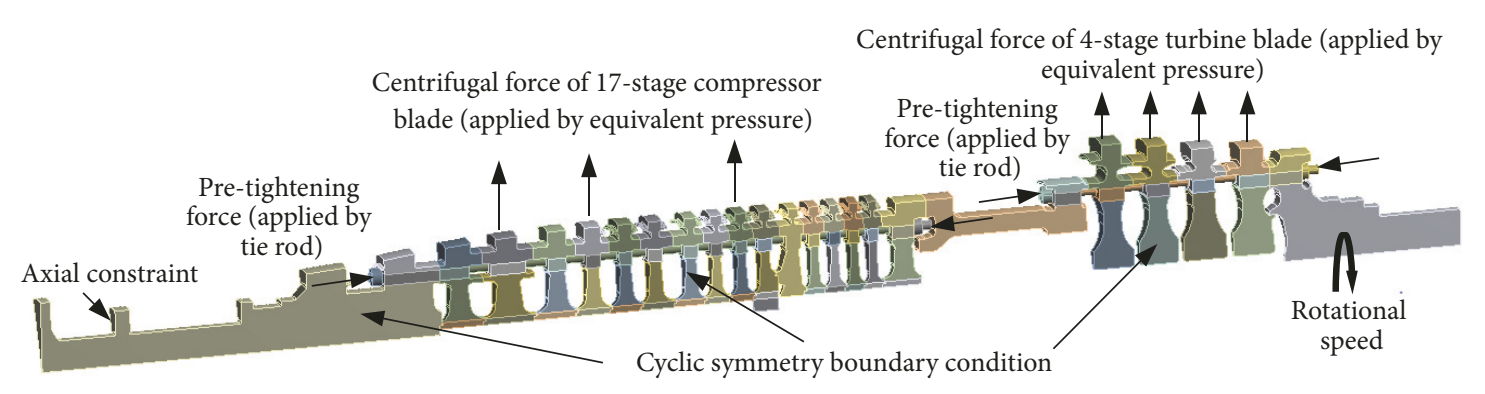

FIGURE 3: Schematic diagram of the boundary conditions and loads of the gas turbine rotor.

and different friction coefficients are given according to the material properties.

The operating environment of the heavy duty gas turbine rotor is high temperature and high load. The requirement of materials of tie rods and turbine discs is rigorous. For the certain gas turbine as the example, the materials of tie rods and turbine discs are high-temperature alloy. The material properties of the rotor used in the research are presented in Tables 1 and 2. For the thermal-structural coupling analysis, the properties under different temperature environment are also listed.

2.3. Loads and Boundary Conditions. According to the operating circumstance of the gas turbine rotor, loads and boundary conditions should be applied on the model, which are shown in Figure 3. To reduce the computational costs, a $1 / 12$ cyclic model is introduced and, therefore, the cyclic symmetry boundary condition is applied on the both sides of the model to simulate the full rotor. The corresponding axial constraint is applied in order to prevent the rigid body motion. There are several methods to apply the pretightening force, including applying force, adopting the preload element PRETS179, applying the equivalent temperature load, and employing contact surface infiltration. In this paper, the pretightening force is applied by adopting the preload element PRETS179. This method could consider the effect of the stretch and shrink of tie rods on the pretightening force, which is produced by the thermal effect and the Poisson effect of discs, so the pretightening force is changing during the calculated time. The centrifugal force of the rotor is applied as the inertial load by setting rotational speed, and the centrifugal force of blades is simulated by applying the pressure on the wheel flange. For the actual gas turbine rotor, the pretightening force is huge, and the effect of the gravity load takes a small proportion in the stress analysis, so the gravity load is not considered in the paper. Considering the temperature load, the transient thermal analysis is carried out to obtain the temperature field, and then the results of transient temperature field are applied as the body load for the structural analysis.

\section{Numerical Results and Analysis}

In this paper, the sequence coupling method is used to analyze the transient stress distribution of the rotor. In general, the sequence coupling method includes two or more analyses arranged in sequence; each of them is attributed to some kinds of physical analysis and they are coupled together by applying the results of one analysis to the next analysis as the load. Thermal-structural coupling analysis 


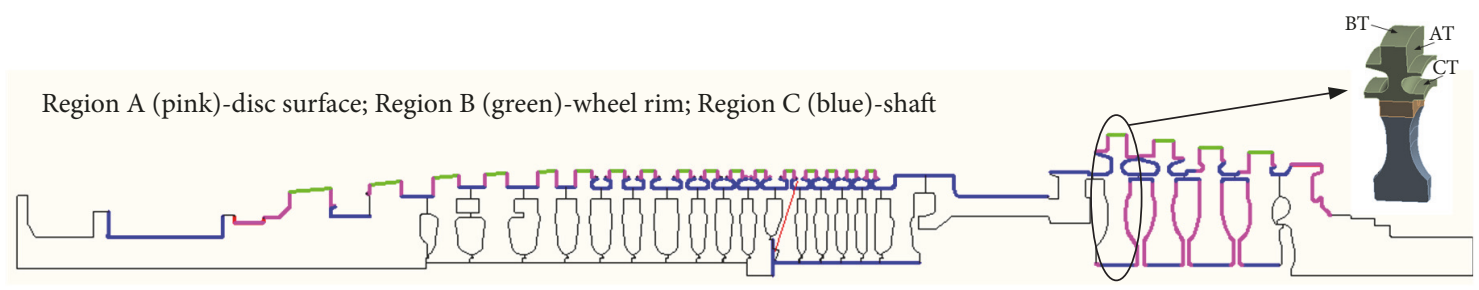

FIGURE 4: Schematic diagram of the thermal boundary conditions.

applies the node temperature obtained from thermal analysis as the thermal load to the later structural analysis according to the start-up sequence. In the paper, the pretightening force is applied on the rods at first. Then thermal analysis is carried out to obtain the temperature distribution of the rotor, which is based on the thermal boundary conditions. Finally, the transient stress analysis is conducted considering the pretightening force, thermal load, and centrifugal load according to the start-up sequence.

\subsection{Analysis of Transient Temperature Distribution}

3.1.1. Thermal Boundary Conditions. The operating condition of heavy duty gas turbine is high temperature. The effect of temperature field on the strength cannot be ignored. The turbine inlet temperature is up to $1400^{\circ} \mathrm{C}$. It must be cooled to ensure that there is no high thermal stress of blade and rotor under the normal working condition. The cooling air of turbine vanes is bled from the appropriate stage of the compressor and is blended into the main airflow after cooling turbine vanes. The cooling air of the turbine rotor and turbine blades is bled from the compressor outlet to cool the rotor and blades through the cooling channel. The high-temperature gas only acts on the turbine blade. Heat is transferred from the blade to the rotor, and the heat is transferred by the conduction method inside the rotor. The rotor is cooled through convection by the cooling air on the rotor surface and in the cooling channel of the rotor. Because the cooling method of gas turbine rotor is convection, it is important to obtain the proper convective heat transfer coefficient. The coefficient is too complex to define, and the theoretical equation of convective heat transfer coefficient cannot be deduced at present; therefore it can only be confirmed by experiment. However, some empirical formulas can be used for reference. In this paper, corresponding empirical formulas are given in different thermal boundary positions of the gas turbine rotor.

All the thermal boundary conditions are determined according to the actual operating conditions of the gas turbine rotor. The temperature and heat transfer coefficient are applied on the rotor as third boundary condition. In Figure 4, three kinds of convective heat transfer regions which are marked by $\mathrm{A}, \mathrm{B}$, and $\mathrm{C}$ are given according to different heat transfer characteristics, where region $\mathrm{A}$ is the thermal boundary condition of the turbine disc surface, region $\mathrm{B}$ represents the thermal boundary condition of the wheel rim, and region $\mathrm{C}$ is the thermal boundary condition of the shaft. Other surfaces were given the adiabatic thermal boundary.
For region A, the empirical formula is given by [22]

$$
h_{\mathrm{A}}=0.03 \frac{\lambda}{R_{\mathrm{A}}}\left(\frac{n \cdot R_{\mathrm{A}}}{v}\right)^{0.8} \text {, }
$$

where $h_{\mathrm{A}}$ is the convective heat transfer coefficient of turbine disc surface, $\lambda$ is the gas thermal conductivity, $R_{\mathrm{A}}$ is the characteristic radius of convective surface, $n$ is the rotating speed, and $v$ is the kinematic viscosity.

For region B, the empirical formula is given by [23]

$$
h_{\mathrm{B}}=\frac{2 \lambda_{b}}{9 \cdot \pi \cdot R_{\mathrm{B}}},
$$

where $h_{\mathrm{B}}$ is the heat transfer coefficient of wheel rim, $\lambda_{b}$ is the material thermal conductivity of blade, and $R_{\mathrm{B}}$ is the characteristic radius of wheel rim. The heat transfer of the wheel rim is mainly affected by the heat conduction.

For region C, the empirical formula is given by [22]

$$
h_{\mathrm{C}}=0.1 \frac{\lambda}{R_{\mathrm{C}}}\left(\frac{n \cdot R_{\mathrm{C}}}{v}\right)^{0.68},
$$

where $h_{\mathrm{C}}$ is the convective heat transfer coefficient of shaft, $\lambda$ is the gas thermal conductivity, $R_{\mathrm{C}}$ is the characteristic radius of shaft, $n$ is the rotating speed, and $v$ is the kinematic viscosity.

To simulate the transient start-up condition, the start-up curve is built up based on the operating data. The operating data includes the rotational speed and the temperature of the location around the 1st turbine wheel rim provided by cooperation with a turbine company. As shown in Figure 5, the rotational speed and temperature arise rapidly during the initial period. The rotational speed reaches the peak of $6000 \mathrm{r} / \mathrm{min}$ at $300 \mathrm{~s}$, and the temperature reaches the peak at $600 \mathrm{~s}$. After the time of $600 \mathrm{~s}$, the gas turbine will run under the steady condition, and the temperature and rotational speed remain constant. To simulate the steady state accurately, the simulation period is set to $6000 \mathrm{~s}$. According to the start-up curve, the start-up time of the gas turbine is short, and large stress will be generated on the gas turbine rotor, so the strength assessment is essential during the startup period.

According to above thermal dynamic theory, the heat transfer coefficients of all surfaces contacting with the gas flow are calculated. The specified values of the thermal boundary conditions including the temperature and pressure are shown in Table 3. Since the amount of the surfaces contacting with the gas flow is large, only the values of 1st 
TABLE 3: The specified values of the thermal boundary conditions.

\begin{tabular}{|c|c|c|c|c|}
\hline \multirow{2}{*}{ Location } & \multicolumn{4}{|c|}{ Time/s } \\
\hline & 150 & 300 & 600 & 6000 \\
\hline \multicolumn{5}{|l|}{ The disc surface of 1st turbine disc (AT) } \\
\hline Temperature of the gas $/{ }^{\circ} \mathrm{C}$ & 172 & 332 & 373 & 373 \\
\hline Pressure of the gas $/ \mathrm{MPa}$ & 0.402 & 0.775 & 0.869 & 0.869 \\
\hline Heat transfer coefficient $h / \mathrm{W} \cdot\left(\mathrm{m}^{-2} \cdot \mathrm{K}^{-1}\right)$ & 556 & 1854 & 2117 & 2117 \\
\hline \multicolumn{5}{|l|}{ The wheel rim of 1st turbine disc (BT) } \\
\hline Temperature of the gas $/{ }^{\circ} \mathrm{C}$ & 231 & 446 & 500 & 500 \\
\hline Pressure of the gas/MPa & 0.542 & 1.051 & 1.183 & 1.183 \\
\hline Heat transfer coefficient $h / \mathrm{W} \cdot\left(\mathrm{m}^{-2} \cdot \mathrm{K}^{-1}\right)$ & 35 & 42 & 51 & 51 \\
\hline \multicolumn{5}{|l|}{ The shaft of 1st turbine disc (CT) } \\
\hline Temperature of the gas $/{ }^{\circ} \mathrm{C}$ & 169 & 327 & 367 & 367 \\
\hline Pressure of the gas/MPa & 0.341 & 0.658 & 0.738 & 0.738 \\
\hline Heat transfer coefficient $h / \mathrm{W} \cdot\left(\mathrm{m}^{-2} \cdot \mathrm{K}^{-1}\right)$ & 468 & 1452 & 1640 & 1640 \\
\hline
\end{tabular}

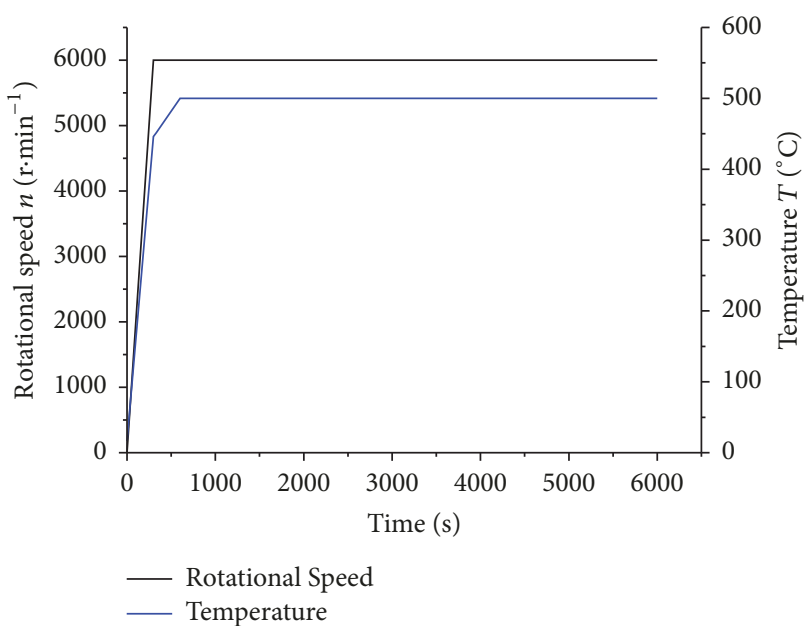

FIGURE 5: The cold start-up curve of the gas turbine rotor.

turbine disc which includes three regions (in Figure 4) are listed.

To reflect the variation tendency of the temperature and stress more distinctly, some special locations of discs should be monitored. The 13th compressor disc and the 1st turbine disc are selected to be monitored objectives. Concrete locations that are monitored are shown in Figure 6. Points A, $\mathrm{B}$, and $\mathrm{C}$ are the locations on the 13th compressor disc, where point $\mathrm{A}$ indicates the location of disc rim; Point $\mathrm{B}$ indicates the location of the tie rod hole; Point $\mathrm{C}$ indicates the location of disc inner margin. Points D, E, and F are the locations on the 1st turbine disc, where point $\mathrm{D}$ indicates the location of disc rim; Point $\mathrm{E}$ indicates the location of the tie rod hole; Point $\mathrm{F}$ indicates the location of disc inner margin.

3.1.2. Temperature Analysis of Gas Turbine Rotor. According to the thermal boundary condition and start-up curve, transient temperature analysis of the rotor has been conducted. Figures 7 and 8 show the temperature distribution for two time points under transient condition. The gradual increase of temperature of the rotor with time can be seen. The temperature increases with the disc diameter in the radial direction, and the temperature level of turbine rotor is higher than that of compressor rotor. During the cold start-up period, the maximum temperature could be observed in the outer surface of the 2nd middle shaft at times of $600 \mathrm{~s}$ and $6000 \mathrm{~s}$. The reason is that the middle shaft is not cooled enough. It also can be seen that the temperature of the 1st turbine disc is high due to the highest inlet temperature.

3.1.3. Temperature Analysis of Monitoring Locations. In order to reflect the temperature variation with time visually, this part gives the temperature of monitoring locations. Figure 9 shows the maximum temperature variation of monitored locations A, B, and C. The temperature difference refers to the difference value between Point A (wheel rim) and Point $\mathrm{C}$ (inner margin). During initial period of start-up condition, the temperature increases rapidly. Then it tends to be stable with the increase of start-up time. The change of temperature difference will reflect the variation of thermal stress. Figure 9 also shows the change tendency of temperature difference. The temperature difference increases rapidly during initial period and then decreases with the time. This indicates that the level of thermal stress will be high at the initial period.

Similar variations of the maximum temperature of the monitored locations $\mathrm{D}, \mathrm{E}$, and $\mathrm{F}$ of the 1st turbine disc can be seen in Figure 10. The temperature difference refers to the difference value between Point $\mathrm{D}$ (wheel rim) and Point F (inner margin). It should be noted that the temperature difference remains constant at the steady period due to the cooling air in the inner margin of disc. By comparing the results in Figures 9 and 10, we can see that the temperature difference of compressor disc is lower than that of turbine disc. The main reason is that there is cooling air in the turbine rotor and the radial temperature gradient is larger on the turbine disc.

3.1.4. Temperature Distribution of the Tie Rod Hole. Since the research is focusing on the tie rod hole, the temperature distribution of two selected holes is illustrated. For the tie rod 


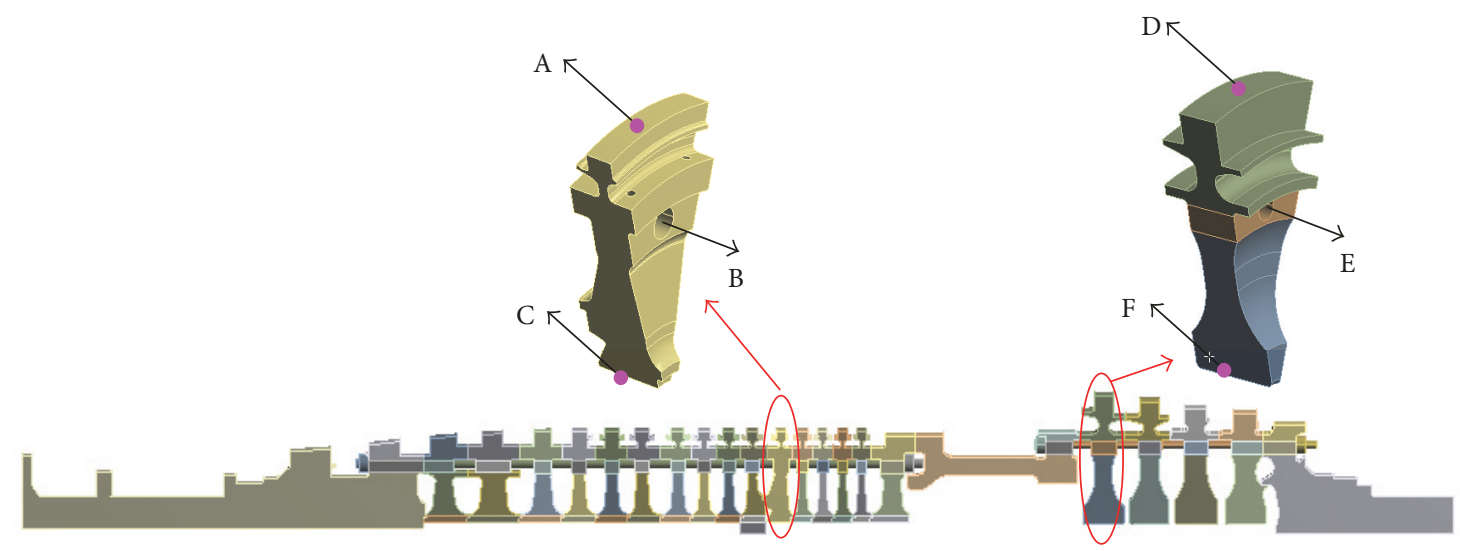

FIGURE 6: Monitoring locations on the gas turbine rotor.

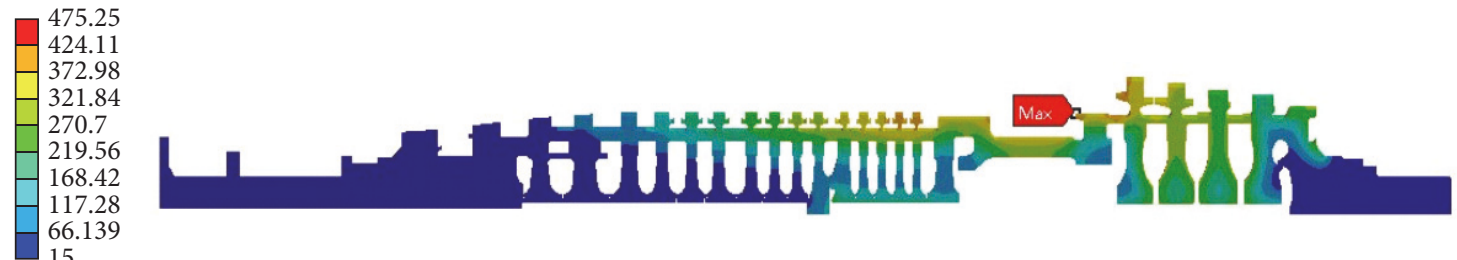

Figure 7: Temperature distribution of the rotor at time of $600 \mathrm{~s} /{ }^{\circ} \mathrm{C}$.

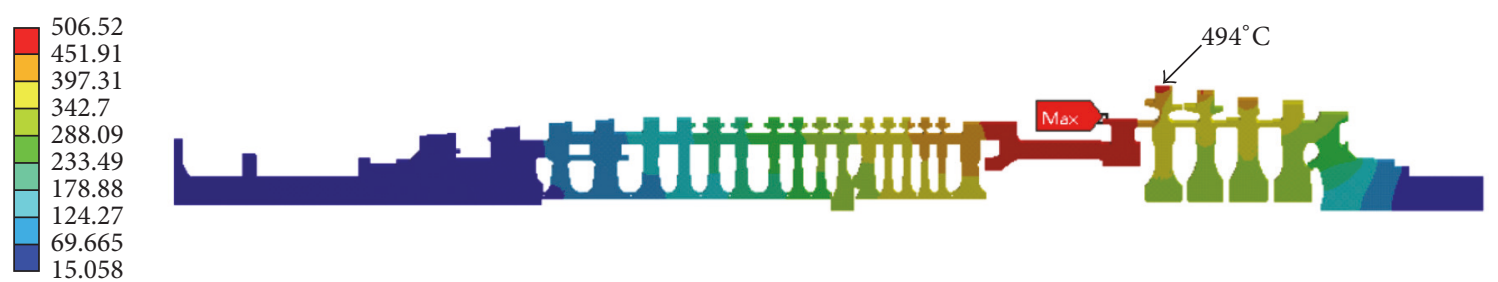

Figure 8: Temperature distribution of the rotor at time of $6000 \mathrm{~s} /{ }^{\circ} \mathrm{C}$.

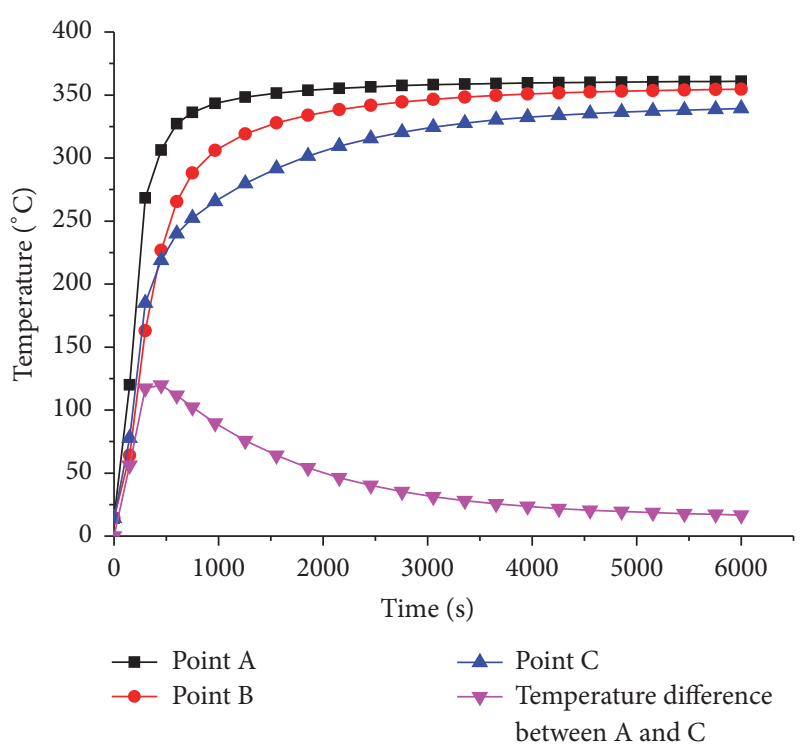

FIGURE 9: Temperature variation of monitored locations on the 13th compressor disc during start-up period.

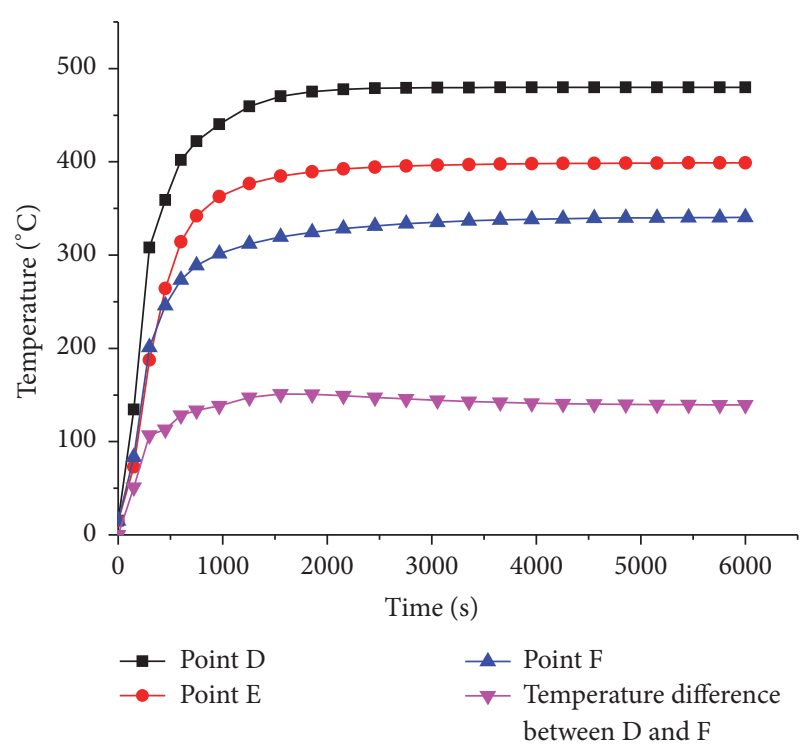

FIGURE 10: Temperature variation of monitored locations on the 1st turbine disc during start-up period. 


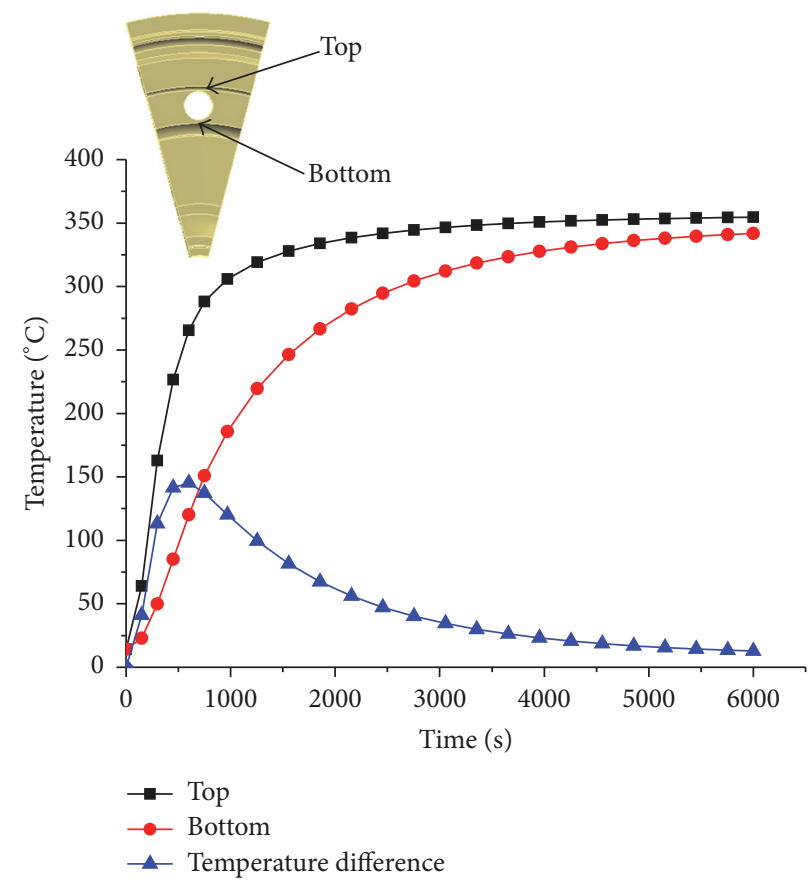

FIGURE 11: Temperature variation of the 13th compressor tie rod hole during start-up period.

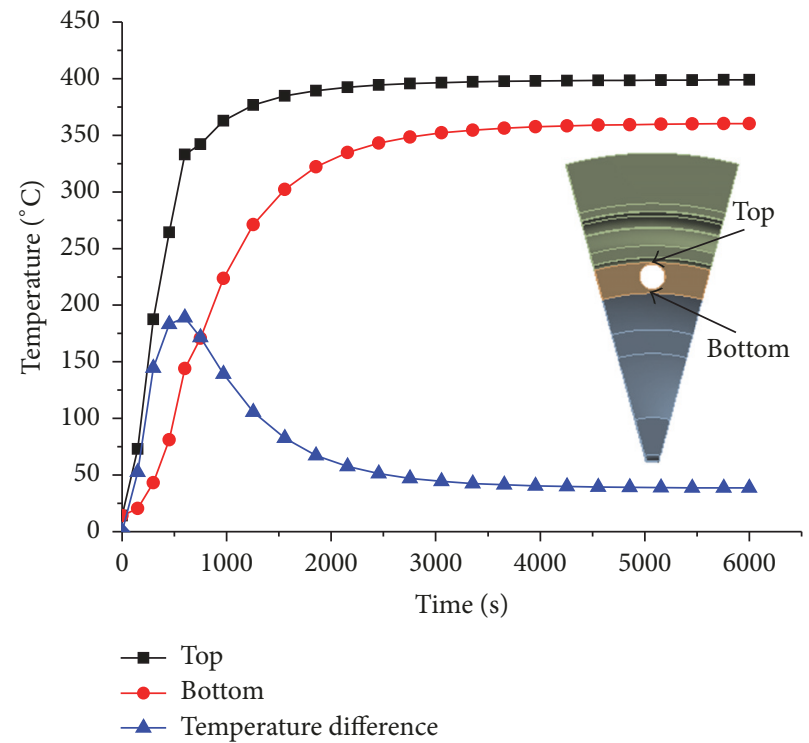

FIGURE 12: Temperature variation of the 1st turbine tie rod hole during start-up period.

hole of 13th compressor disc, the variation of the maximum temperature on the top and bottom is shown in Figure 11. The temperature difference refers to the difference value between top and bottom in this figure. The temperature increases rapidly in the top, whereas it increases slowly in the bottom. The reason is that the heat needs more time to be transferred from the wheel rim to the inner margin. The magnitude of the temperature difference, which represents the temperature gradient of the disc hole, increases till a peak at $600 \mathrm{~s}$ and then decreases slowly with the increase of time. Thus, the maximum temperature gradient of the hole occurs at $600 \mathrm{~s}$.

Similar temperature variation of the 1st turbine disc tie rod hole is shown in Figure 12. The maximum temperature gradient of the hole occurs at time of $600 \mathrm{~s}$ as well. It should be noted that the magnitude of corresponding gradient of the turbine disc is higher than that of the compressor generally, which indicates that the effect of thermal stress on the turbine tie rod hole is greater. 


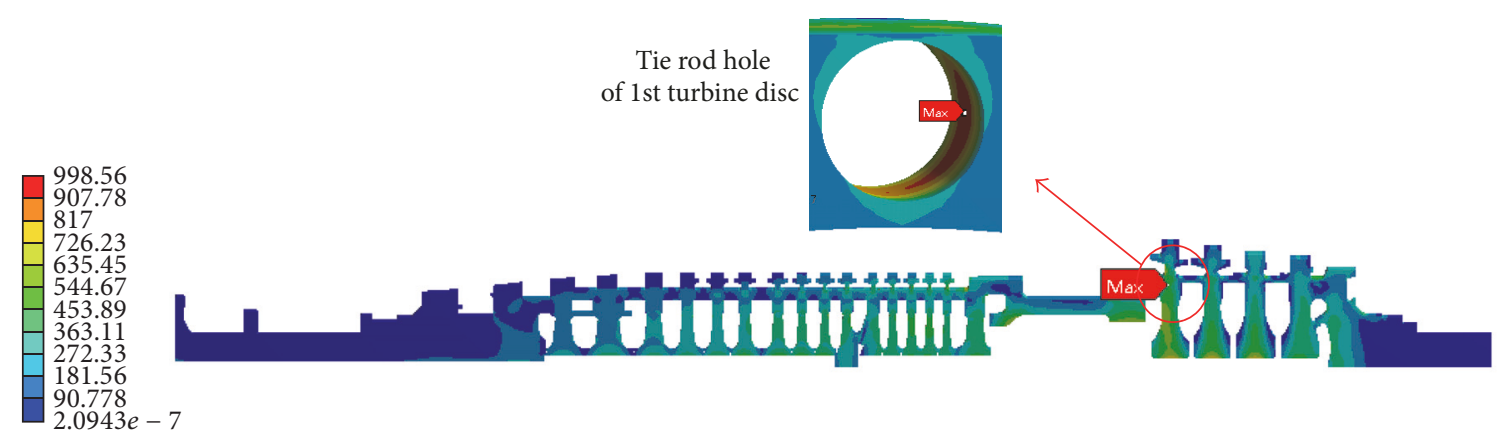

FIgURE 13: Equivalent stress distribution of the rotor at time of $600 \mathrm{~s} / \mathrm{MPa}$.

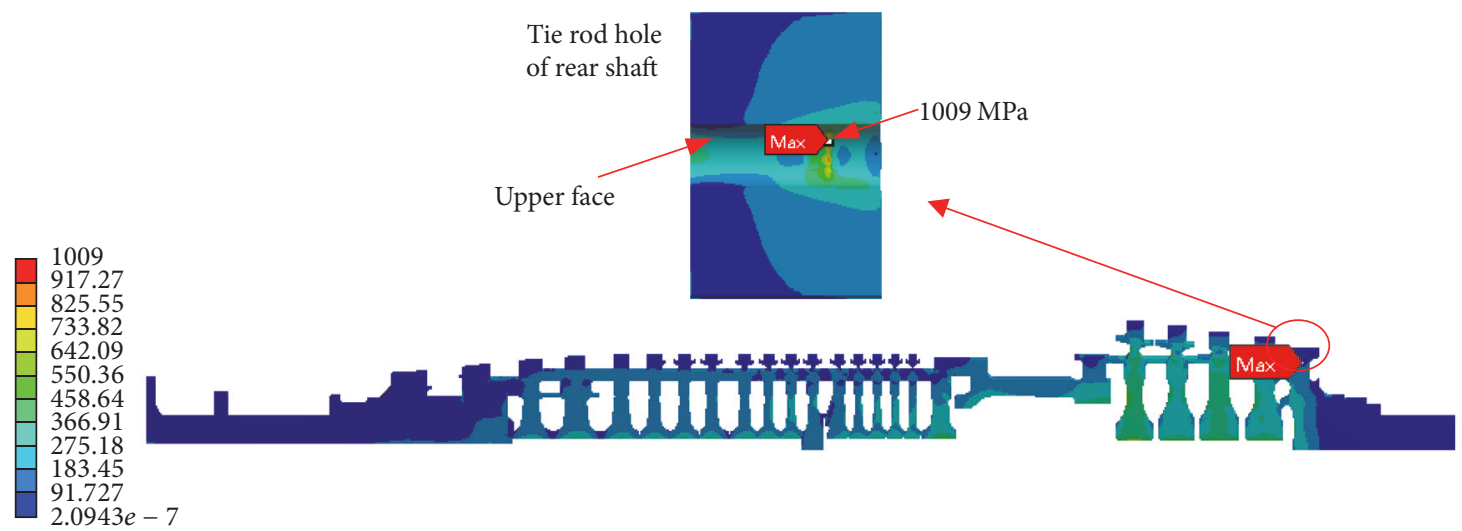

FIGURE 14: Equivalent stress distribution of the rotor at time of $6000 \mathrm{~s} / \mathrm{MPa}$.

3.2. Analysis of Transient Equivalent Stress Distribution. In this section, the equivalent stress variation of the rotor will be shown. Firstly, the stress distributions of the whole rotor at different time are given. Secondly, the variation tendency of the maximum equivalent stress at the rear shaft tie rod hole is shown in detail. Thirdly, the change tendency of the tightening force of tie rods is illustrated. Finally, the maximum equivalent stress of the monitoring locations at different time is shown.

3.2.1. Transient Equivalent Stress Distribution of the Gas Turbine Rotor. Figures 13 and 14 show the stress distribution at $600 \mathrm{~s}$ and $6000 \mathrm{~s}$ during start-up period. At time of $600 \mathrm{~s}$, the maximum equivalent stress could be observed in the side face of 1st turbine disc hole. The maximum equivalent stress occurs in the upper face of the rear shaft hole at time of 6000 s. According to the stress distribution, the high-stress areas are mainly concentrated in the turbine rotor. The stress level of compressor rotor is low. During the start-up period, the maximum equivalent stress occurs at the rear shaft hole. It should be noted that the stress distribution of the rotor at $6000 \mathrm{~s}$ is similar to that at steady-state condition. Since the gas turbine rotor is running in steady-state condition at most of the time, the maximum equivalent stress at $6000 \mathrm{~s}$ has significant impact on the strength of the rotor. The design improvement is introduced to reduce the stress in the rest of the paper.
3.2.2. Transient Equivalent Stress Distribution of the Tie Rod Hole. Since the high stress region occurs at the rear shaft hole, the detailed introduction of the stress distribution is given in this part. In Figure 15, the variation of the equivalent stress at the location of the rear shaft tie rod hole is shown, and the change tendency of contact pressure between the tie rod shoulder and hole is also illustrated. As shown in Figure 16, different locations of the maximum equivalent stress on the tie rod hole during the start-up period are given in detail. The results indicate that the location of maximum equivalent stress is variable under start-up condition. In addition, the magnitude of the maximum equivalent stress increases with time till reaching a peak. A negligible change in the maximum equivalent stress can be observed with the further increase of time. To interpret the phenomenon, the whole start-up period is divided into 4 steps: step 1 from time of $0 \mathrm{~s}$ to $300 \mathrm{~s}$, step 2 from time of $300 \mathrm{~s}$ to $600 \mathrm{~s}$, step 3 from time of $600 \mathrm{~s}$ to $1800 \mathrm{~s}$, and step 4 from time of $1800 \mathrm{~s}$ to $6000 \mathrm{~s}$.

During step 1, the rotor is operated under low load and the rotational speed is low, the tie rod shoulder and corresponding hole have not contacted each other, and the contact pressure is 0 . Meanwhile, the circular hole starts to transform into an oval one, whose deformation is generated by the rotational speed almost. Therefore, the hole deformation determines the location and magnitude of the maximum equivalent stress in this period. During step 2, the upper face of the shoulder begins to contact with the 


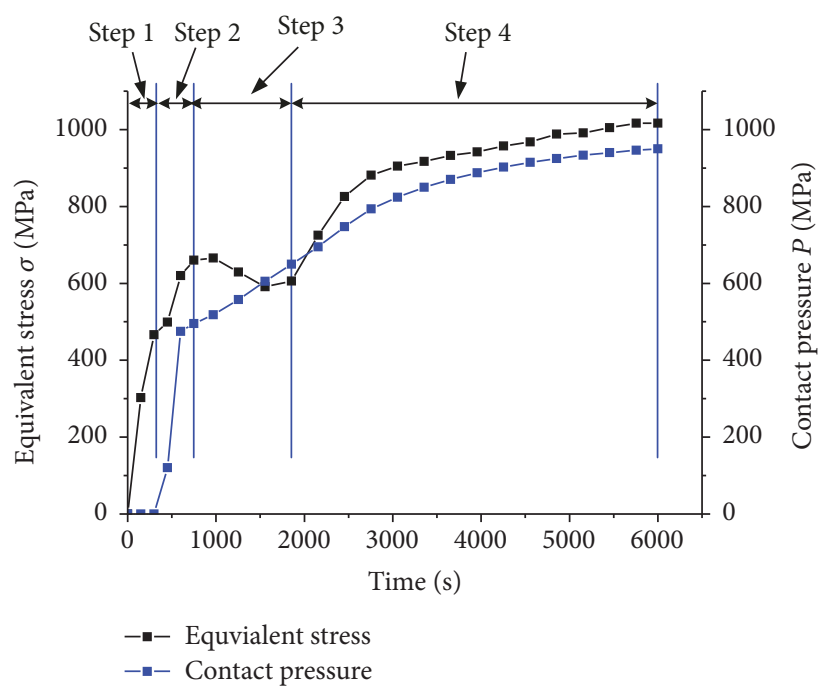

FIGURE 15: The variation of the maximum equivalent stress and contact pressure of the rear shaft hole during the start-up period.

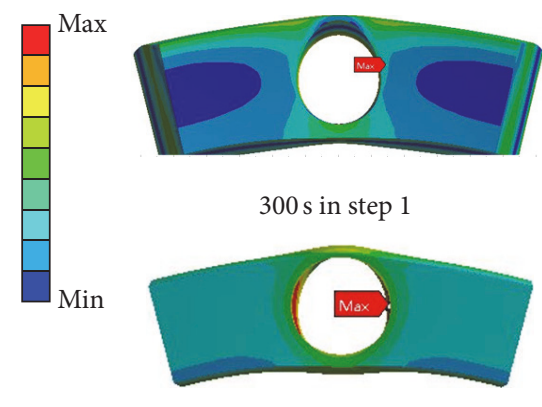

$1800 \mathrm{~s}$ in step 3

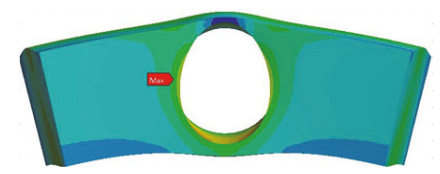

$600 \mathrm{~s}$ in step 2

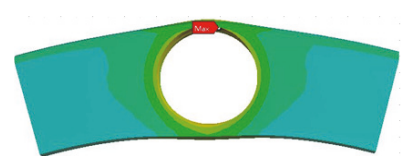

$6000 \mathrm{~s}$ in step 4

FIGURE 16: The variation of the maximum equivalent stress location of the rear shaft hole during the start-up period.

hole, and the contact pressure increases with the time. Due to the rising temperature gradient, the deformation of the hole becomes larger and reaches the peak along with the temperature gradient at time of $600 \mathrm{~s}$. Because the contact between shoulders and holes is inadequate, the location and magnitude of the maximum equivalent stress are still determined by the hole deformation. During step 3, the contact pressure becomes lager and the oval hole starts to transform back into a circular one as the temperature gradient decreases. However, the impact of the contact pressure is still weaker than that of the hole deformation. During step 4, since the temperature distribution becomes steady, the oval hole transforms back into a circular one completely and the contact pressure determines the magnitude of the maximum equivalent stress in this period. The contact pressure and the maximum equivalent stress increase with time under constant rotational speed.

The maximum equivalent stress of the rotor occurs on the upper face of the rear shaft hole at time of $6000 \mathrm{~s}$. The main reason is the contact pressure between the tie rod shoulder and hole. Therefore the design improvement of the tie rod is introduced according to the above analysis.
3.2.3. Transient Equivalent Stress Distribution of the Tie Rod. Because the huge pretightening force, rotational speed, and thermal load are applied on the tie rod, the tensile deformation and contact extrusion deformation which will result in the large stress are produced. The stress level of the tie rod is also the focus in the analysis. The variation of the tightening force and maximum equivalent stress of the compressor tie rod are shown in Figure 17. The magnitude of the maximum equivalent stress and pretightening force increase till reaching the peak and then decrease slowly with the increase of time. Thus, the pretightening force and the maximum equivalent stress both reach the peak at $970 \mathrm{~s}$. The equivalent stress distribution at $970 \mathrm{~s}$ is shown in Figure 18. The maximum stress occurs at the shoulder fillet. Due to the rising temperature of the tie rod and larger thermal expansion coefficient of the material, the tie rod expands over time, whereas the axial expansion of the rotor is steady with the increase of time. The tie rod becomes slacker and the pretightening force and maximum equivalent stress decrease with the increase of time.

Similar variation of the pretightening force and the maximum equivalent stress of the turbine tie rod are shown 


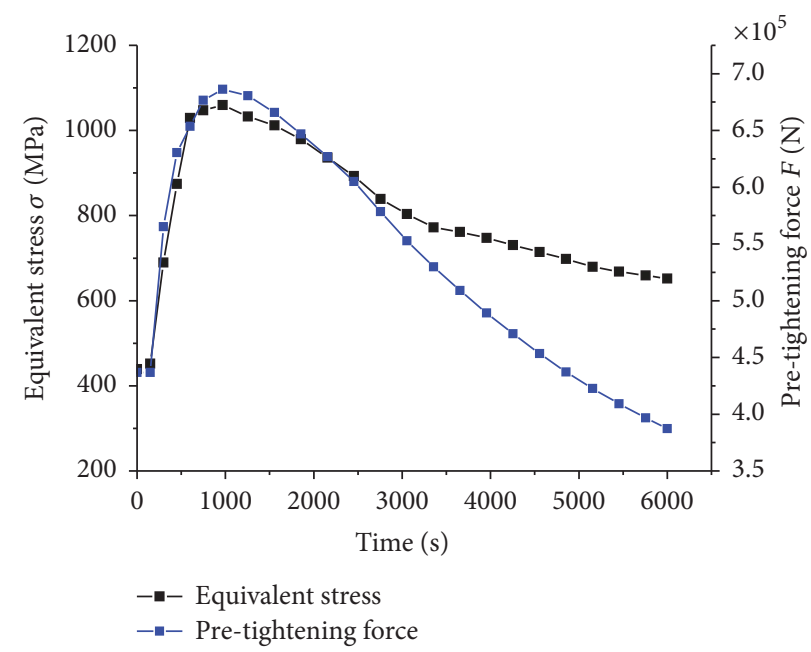

FIGURE 17: The variation of the maximum equivalent stress and pretightening force of the compressor tie rod during start-up period.

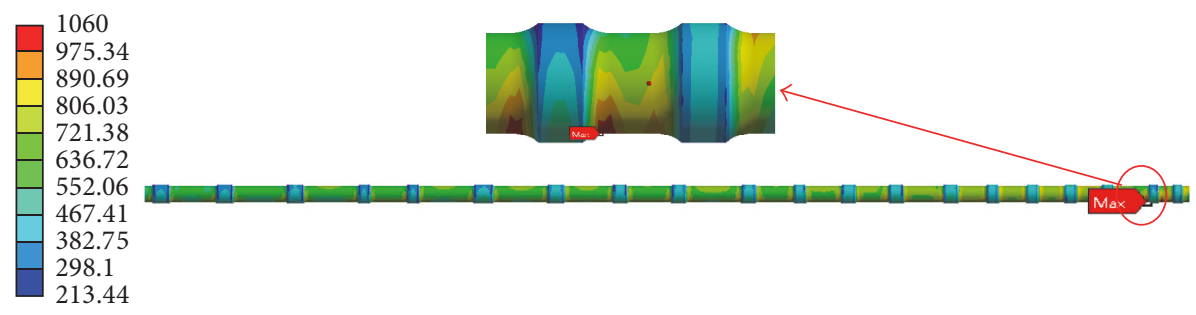

FIGURE 18: Equivalent stress distribution of the compressor tie rod at time of $970 \mathrm{~s} / \mathrm{MPa}$.

in Figure 19. Compared with the compressor tie rod, the temperature of the turbine tie rod is higher; the expansion and corresponding stress response are more positive as well. Thus, the peak time of the tightening force and equivalent stress are brought forward to time of $600 \mathrm{~s}$. The equivalent stress distribution at $600 \mathrm{~s}$ is shown in Figure 20. The maximum equivalent stress occurring at the shoulder fillet is $1287 \mathrm{MPa}$. Meanwhile, the stress value of the shoulder fillet is maximum for the gas turbine rotor during the start-up period. Since the contraction ratio of the turbine tie rod is larger than that of the compressor tie rod, the change tendency of the pretightening force of the turbine tie rod is more violent than that of the compressor tie rod. Moreover, because of the unique structure at tie rod end of right hand, the maximum equivalent stress of the turbine tie rod remains at a higher level than that of the compressor tie rod.

3.2.4. Transient Equivalent Stress Distribution of Monitoring Locations. The variations of the maximum stress of the 13th compressor disc monitored locations are shown in Figure 21. It could be observed that the stress change tendencies of different monitoring points are similar. During initial period, the maximum equivalent stress increases rapidly with rotational speed and thermal load and then decreases gradually as the temperature gradient becomes stable at steady period. As shown in Figure 21, the stress levels of the three monitoring points $\mathrm{A}, \mathrm{B}$, and $\mathrm{C}$ are basically the same at the initial start-up period; the difference comes from the effect of rotational speed. As the time increases, the temperature gradient increases and the thermal stress begins to dominate. Since the temperature gradient of Point $\mathrm{A}$ is less than that of Point $C$, the equivalent stress of Point $A$ is lower than that of Point $\mathrm{C}$. The stress concentration is prone to occur at Point $\mathrm{B}$ due to the structure of tie rod hole. The maximum equivalent stress of the 13th compressor disc occurs in the tie rod hole at time of $600 \mathrm{~s}$.

Similar variations of the equivalent stress of the 1st turbine disc monitored locations are shown in Figure 22. It should be noted that the equivalent stress of Point $\mathrm{E}$ is largest. At three points, as the time increases, the equivalent stress increases rapidly and then decreases to the steady value. The equivalent stress level of Point D is still lower than that of Point F due to the temperature gradient. The stress concentration is prone to occur in location $\mathrm{E}$, and the maximum equivalent stress of the 1st turbine disc occurs in the tie rod hole at time of $600 \mathrm{~s}$.

3.3. Structural Design Improvement of the Turbine Tie Rod. According to above analysis results, the dangerous locations during the cold start-up period occur on the turbine tie rod hole and corresponding tie rod. The main reason is the compressional deformation between the tie rod shoulder and hole. Therefore, the tie rod structure is the objective to be improved. In this paper, the novel tie rod structure is introduced to reduce the equivalent stress. Figure 23 shows two kinds of tie rod structure. For structure A, there is only one tie rod shoulder near the right nut. For the novel structure 


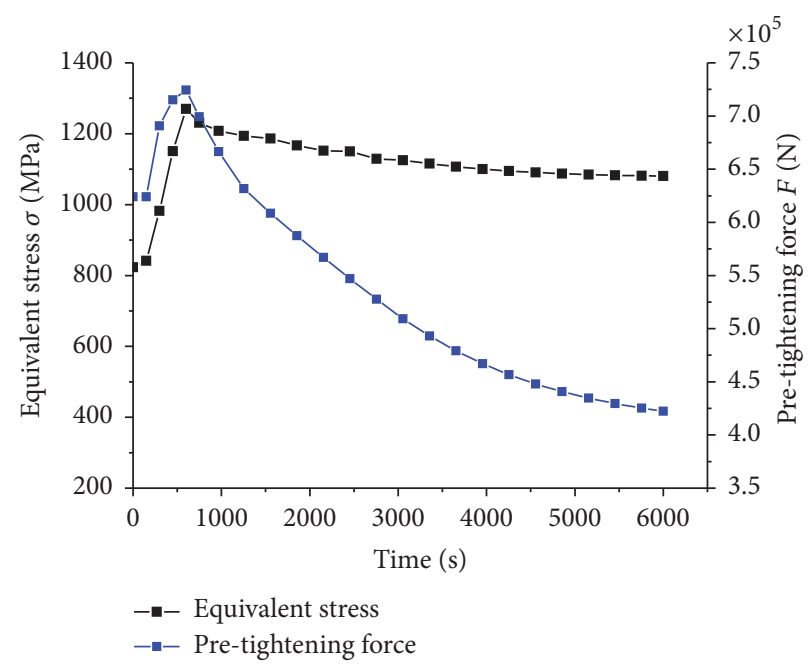

FIGURE 19: The variation of the maximum equivalent stress and pretightening force of the turbine tie rod.

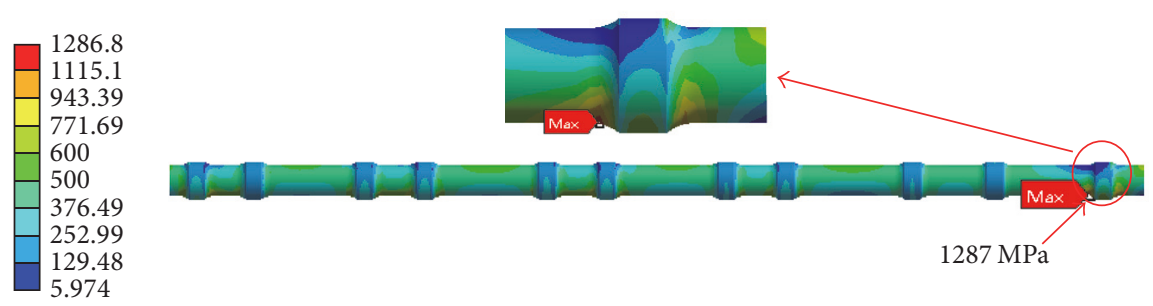

FIGURE 20: Equivalent stress distribution of the turbine tie rod at time of $600 \mathrm{~s} / \mathrm{MPa}$.

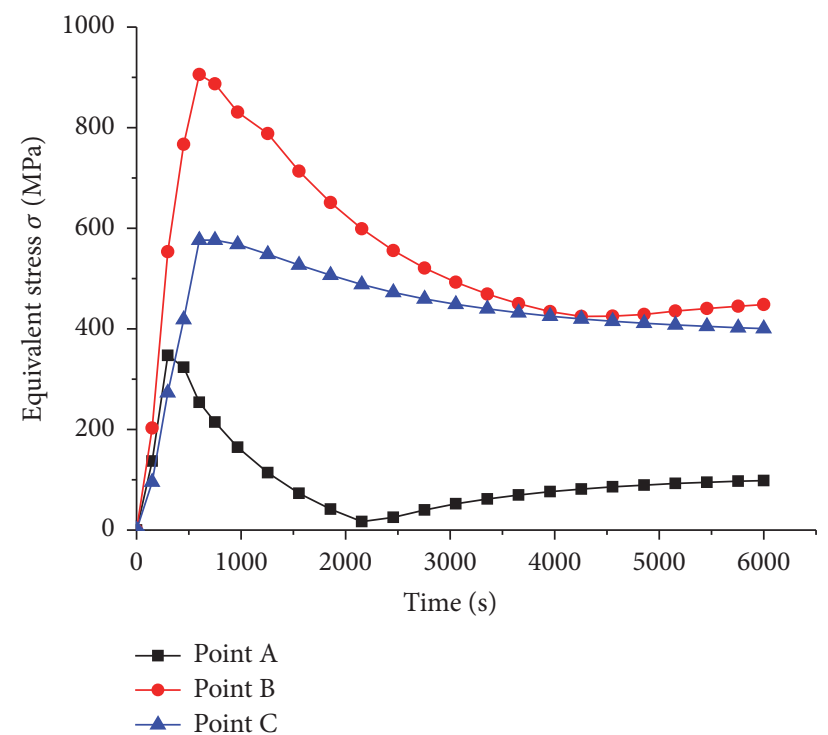

FIGURE 21: The equivalent stress variation of the compressor monitoring locations during start-up period.

$B$, two tie rod shoulders are introduced near the right nut. Compared with structure A, structure B can provide the twopoint support to reduce the contact pressure between the shoulder and hole. Structure B also ensures that each tie

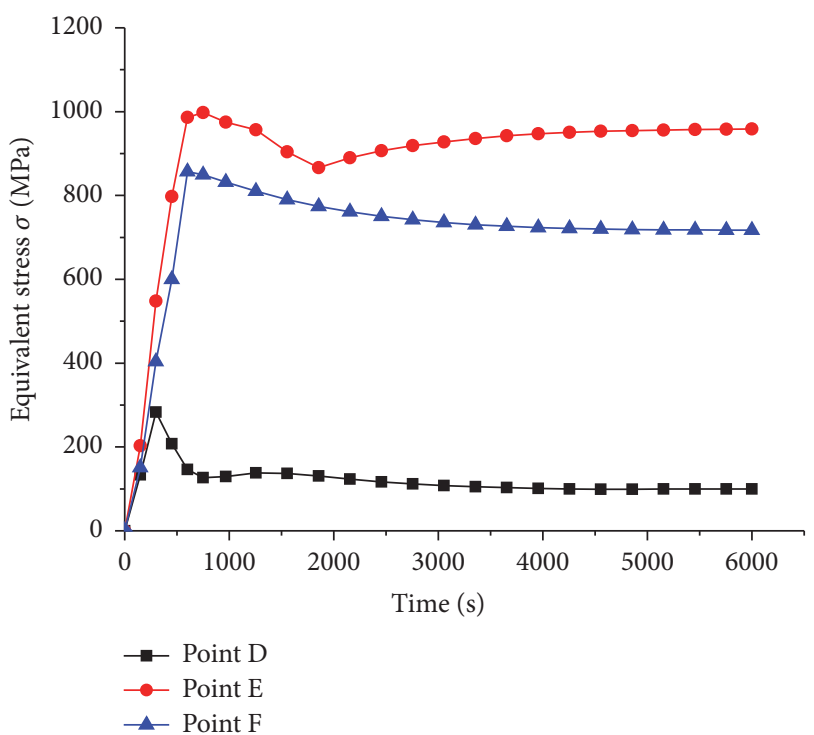

FIGURE 22: The equivalent stress variation of the turbine monitoring locations during start-up period.

rod hole corresponds to double shoulders. For the double shoulder structure, the contact pressure on the single shoulder is reduced, and the compressional deformation between the shoulder and hole decreases with the contact pressure. 

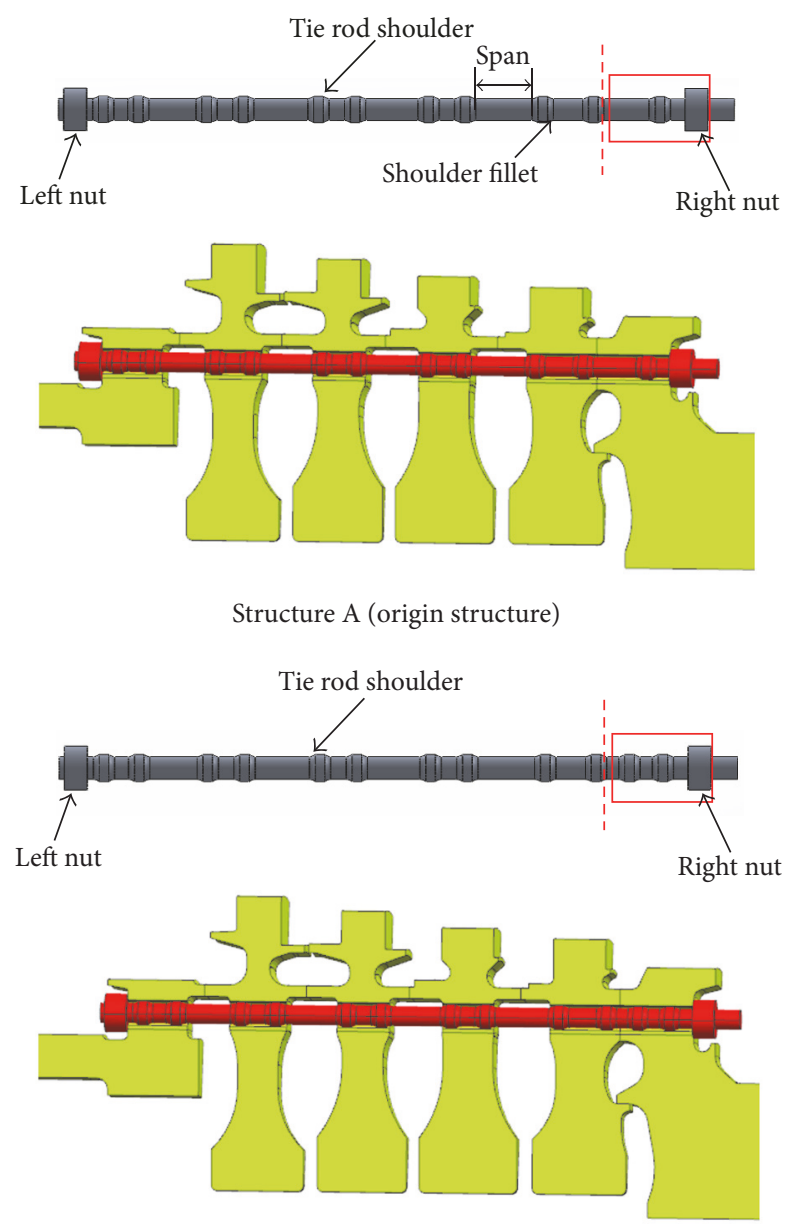

Structure B (novel structure)

FIgURE 23: Two kinds of turbine tie rod structure.

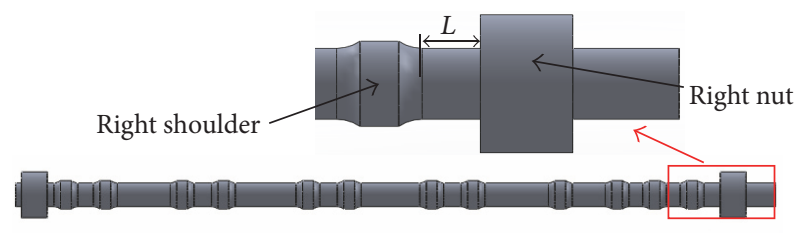

FIgURE 24: The span between shoulder and nut.

As the compressional deformation mainly contributes to the equivalent stress, the maximum equivalent stress of the tie rod hole also decreases. At the same time, the double shoulder structure reduces the span between two shoulders; it is beneficial to lower the equivalent stress level at the shoulder fillet.

Meanwhile, the distance $L$ between the right shoulder and right nut has significant effect on the equivalent stress. Figure 24 shows the span distance $L$. The stress results under different span distances are shown in Figure 25. The initial span distance $L$ is $14 \mathrm{~mm}$. Considering the structure of the tie rod, the optimal value of $L$ is $10 \mathrm{~mm}$. When the distance is less than $10 \mathrm{~mm}$, the equivalent stress concentration at the
TABLE 4: The decrease ratio of maximum equivalent stress after design improvement.

\begin{tabular}{lccc}
\hline & & $\begin{array}{c}\text { Tie rod hole of } \\
\text { rear shaft }\end{array}$ & Turbine tie rod \\
\hline $\begin{array}{l}\text { Maximum } \\
\text { equivalent } \\
\text { stress/MPa }\end{array}$ & $\begin{array}{c}\text { Before design } \\
\text { improvement } \\
\text { After design } \\
\text { improvement }\end{array}$ & 1009 & 1287 \\
Decrease ratio/\% & 761 & 1088 \\
\hline
\end{tabular}

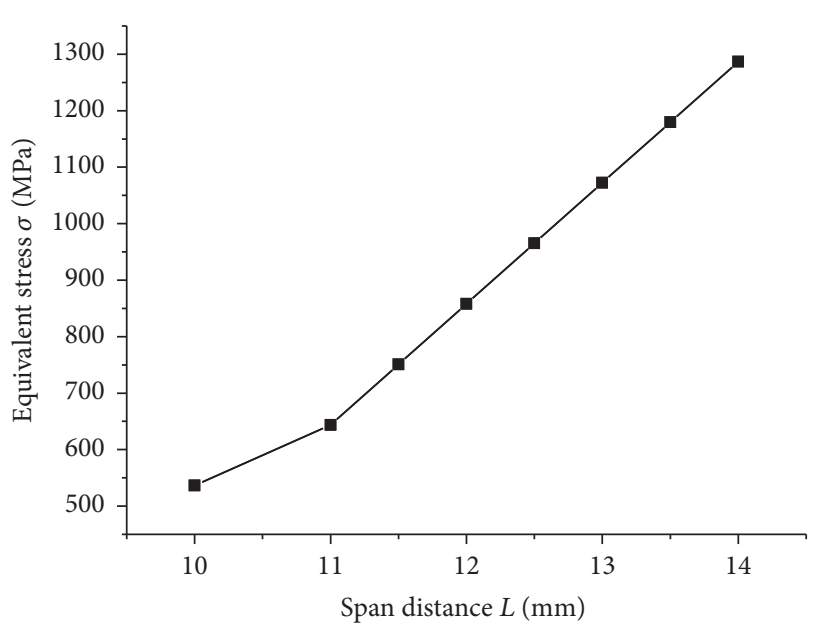

FIgURE 25: The results under different span distances.

right nut cannot be released and there will be a large stress region occurring at the right nut. According to above analysis results, the novel tie rod structure is shown in Figure 26. For the novel structure, the double-shoulder structure is applied and the distance $L$ is $10 \mathrm{~mm}$. Table 4 shows the stress results after applying this novel tie rod structure. The maximum equivalent stress of the rotor decreases obviously.

\section{Conclusion}

In this paper, three-dimensional finite element analysis is conducted to identify the temperature and equivalent stress distribution of the gas turbine rotor under cold start-up condition. The temperature and stress distributions of the rotor are shown in detail. A novel tie rod structure is introduced to reduce the maximum equivalent stress. The results are summarized below.

(1) The temperature field of the rotor is obtained according to the thermal dynamic theory. The temperature changes obviously along the radial and axial direction. The temperature field of the compressor rotor increases gradually along the axial direction, and the maximum temperature appears on the 17th stage. The radial temperature gradient of the turbine rotor is obvious. The highest temperature of the turbine rotor occurs at the 1st stage wheel rim.

(2) During the cold start-up period, the maximum equivalent stress location of the tie rod hole is variable. The magnitude of the maximum equivalent stress increases with 


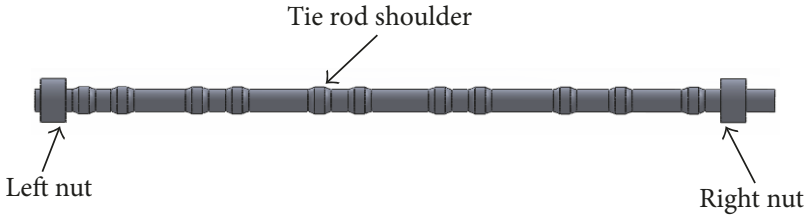

(a) Original structure

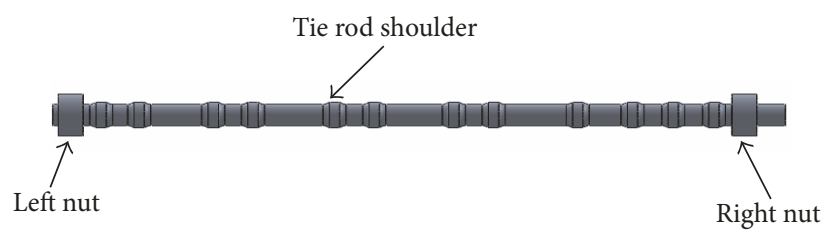

(b) Novel structure

FIGURE 26: The novel tie rod structure.

time till reaching a peak and then decreases to a steady value. Because of the unique tie rod structure, the equivalent stress concentration is prone to occur at the tie rod and tie rod hole of the rear shaft during the cold start-up period. The whole start-up period is divided into 4 steps according to the deformation law and contact behavior of tie rod hole. The maximum equivalent stress of the tie rod hole occurs at the 4 th step due to the contribution of contact pressure.

(3) The maximum equivalent stress of the rotor occurs at the turbine tie rod during the start-up period. The change tendency of the maximum equivalent stress is consistent with that of the pretightening force. The maximum equivalent stress and pretightening force increase till reaching a peak and then decrease slowly with the increase of time during the cold start-up period. It should be noted that the pretightening force increases till reaching a peak and then drops to the steady value during the start-up period. Because the thermal expansion of the disc is larger than that of tie rod, the maximum pretightening force is larger than the initial pretightening force.

(4) A novel tie rod structure is introduced to reduce the equivalent stress level of tie rod hole. Double tie rod shoulders are applied near the right nut in the novel structure. The structure can provide the two-point support to reduce the contact pressure between the shoulder and hole. It also ensures that each tie rod hole corresponds to double shoulders. Meanwhile, reducing the distance $L$ between the right shoulder and right nut has significant effect on the equivalent stress. The results can provide some reference for the design of the gas turbine rotor.

\section{Conflicts of Interest}

The authors declare that there are no conflicts of interest regarding the publication of this paper.

\section{Acknowledgments}

This work is supported by the National Natural Science Foundation of China (no. 11372234) and the Key Project of Natural Science Foundation of Xi'an Jiaotong University (no. $\operatorname{zrzd} 2017025)$.

\section{References}

[1] H.-G. Li, H. Liu, and L. Yu, "Determination of preload force of circumferential distributed rod fastening rotor," Hangkong Dongli Xuebao/Journal of Aerospace Power, vol. 26, no. 12, pp. 2791-2797, 2011.
[2] X. Liu, Q. Yuan, and W. Ou, "Strength analysis and design improvement of the tie rods in circumferentially distributed rod fastening rotors," Xi'an Jiaotong Daxue Xuebao/Journal of Xi'an Jiaotong University, vol. 50, no. 10, pp. 104-110, 2016.

[3] R. Zhu, G. Meng, H. G. Li et al., "Transient characteristics analysis of thermal stress in start-stop condition for rotor system in gas turbine," Advances in vibration engineering, vol. 12 , no. 6, pp. 571-586, 2013.

[4] C. Zhang and Z. L. Xu, "Steam Turbine Rotor Thermal Stress Calculation with Thermo-Structure Coupled Model," Journal of Xian Jiaotong university, vol. 48, no. 4, pp. 68-72, 2014.

[5] P. S. Das, "Three-dimensional structural evaluation of a gas turbine engine rotor," in Proceedings of the ASME Turbo Expo 2014: Turbine Technical Conference and Exposition, GT 2014, Germany, June 2014.

[6] M. J. Janssen and J. S. Joyce, "35-year old splined-disc rotor design for large gas turbines," in Proceedings of the ASME 1996 International Gas Turbine and Aeroengine Congress and Exhibition, GT 1996, UK, June 1996.

[7] S.-X. Yuan, Y.-Y. Zhang, Y.-C. Zhang, and X.-J. Jiang, "Stress distribution and contact status analysis of a bolted rotor with curvic couplings," Proceedings of the Institution of Mechanical Engineers, Part C: Journal of Mechanical Engineering Science, vol. 224, no. 9, pp. 1815-1829, 2010.

[8] A. Rusin, G. Nowak, and W. Piecha, "Shrink connection modelling of the steam turbine rotor," Engineering Failure Analysis, vol. 34, pp. 217-227, 2013.

[9] F. Lanoue, A. Vadean, and B. Sanschagrin, "Finite element analysis and contact modelling considerations of interference fits for fretting fatigue strength calculations," Simulation Modelling Practice and Theory, vol. 17, no. 10, pp. 1587-1602, 2009.

[10] R. A. Claudio, C. M. Branco, E. C. Gomes, G. F. Harrison, and M. R. Winstone, "Fatigue life prediction and failure analysis of a gas turbine disc using the finite-element method," Fatigue fracture of engineering materials structures, vol. 27, no. 9, pp. 849-860, 2004.

[11] S. Barella, M. Bellogini, M. Boniardi, and S. Cincera, "Failure analysis of a steam turbine rotor," Engineering Failure Analysis, vol. 18, no. 6, pp. 1511-1519, 2011.

[12] G. Song, B. Kim, and S. Chang, "Fatigue life evaluation for turbine rotor using green's function," Procedia Engineering, vol. 10, pp. 2292-2297, 2011.

[13] X. P. Li, A. L. Wang, and S. X. Gao, "Fatigue damage research on rod-fastening rotor of gas turbine considering start-up stage," Applied Mechanics and Materials, vol. 387, pp. 221-226, 2013.

[14] Q. L. Zhang and W. P. Huang, "Research on the Fatigue Life of Circumferential Distributed Rod Fastening Rotor," Machine Design Manufacture, vol. 1, pp. 238-242, 2016.

[15] C. Zhang, N. Hu, J. Wang, Q. Chen, F. He, and X. Wang, "Thermal stress analysis for rotor of $600 \mathrm{MW}$ supercritical steam 
turbine," in Proceedings of the 2011 International Conference on Consumer Electronics, Communications and Networks, CECNet 2011, pp. 2824-2827, China, April 2011.

[16] X. Hai-jun and S. Jian, "Failure analysis and optimization design of a centrifuge rotor," Engineering Failure Analysis, vol. 14, no. 1, pp. 101-109, 2007.

[17] Y. Li, H. Sun, and Y. Nie, "Thermal stress analysis of 600MW steam turbine rotor in different governing modes," in Proceedings of the Asia-Pacific Power and Energy Engineering Conference, APPEEC 2010, China, March 2010.

[18] A. Nayebi, H. Ranjbar, and H. Rokhgireh, "Analysis of unified continuum damage mechanics model of gas turbine rotor steel: Life assessment," Proceedings of the Institution of Mechanical Engineers, Part L: Journal of Materials: Design and Applications, vol. 227, no. 3, pp. 216-225, 2013.

[19] Y. Fukuda, "Development of advanced ultra supercritical fossil power plants in Japan: Materials and high temperature corrosion properties," Materials Science Forum, vol. 696, pp. 236-241, 2011.

[20] G. Nowak and A. Rusin, "Shape and operation optimisation of a supercritical steam turbine rotor," Energy Conversion and Management, vol. 74, pp. 417-425, 2013.

[21] Y. Liu, Q. Yuan, and Z. Zhou, "Contact status analysis of rod-fastened rotors with hirth coupling in gas turbines," in Proceedings of the ASME Turbo Expo 2015: Turbine Technical Conference and Exposition, GT 2015, Canada, June 2015.

[22] C. Y. Li and Y. C. Zhang, "Calculation and Analysis of the Transient Three-dimensional Temperature and Stress Field of a Gas Turbines Disk," Journal of Power Engineering, vol. 2, no. 26, pp. 211-214, 2006.

[23] J. Y. Shi and Z. C. Deng, "Heat Transfer Coefficient of Wheel Rims of Large Capacity Steam Turbines," Journal of Power Engineering, vol. 2, no. 27, pp. 153-156, 2007. 


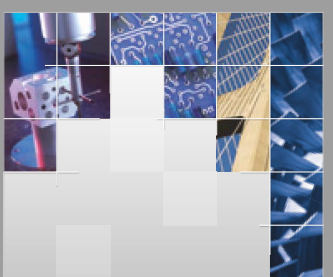

\section{Enfincering}
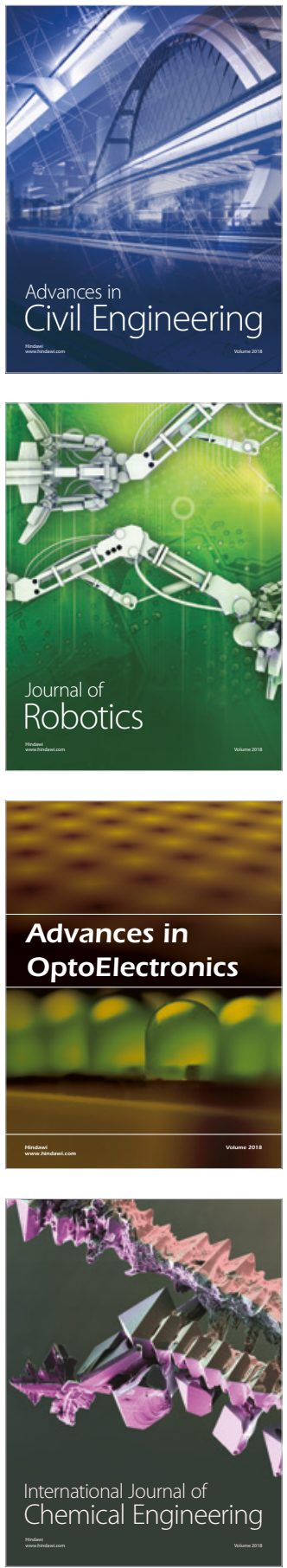

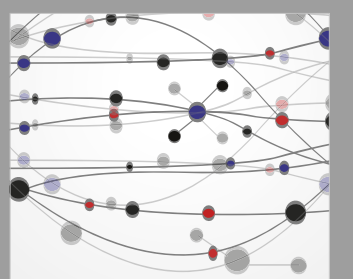

\section{Rotating \\ Machinery}

The Scientific World Journal

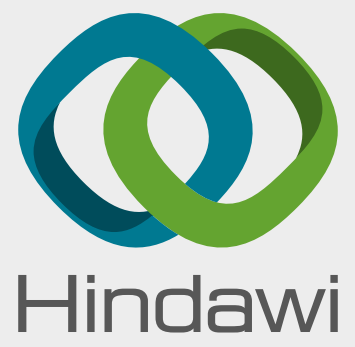

Submit your manuscripts at

www.hindawi.com
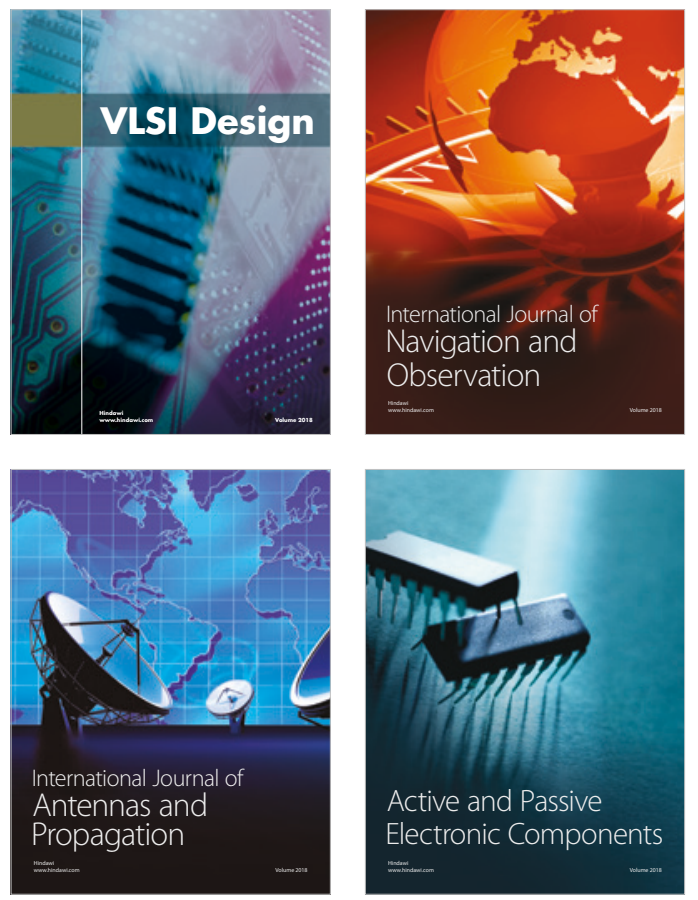
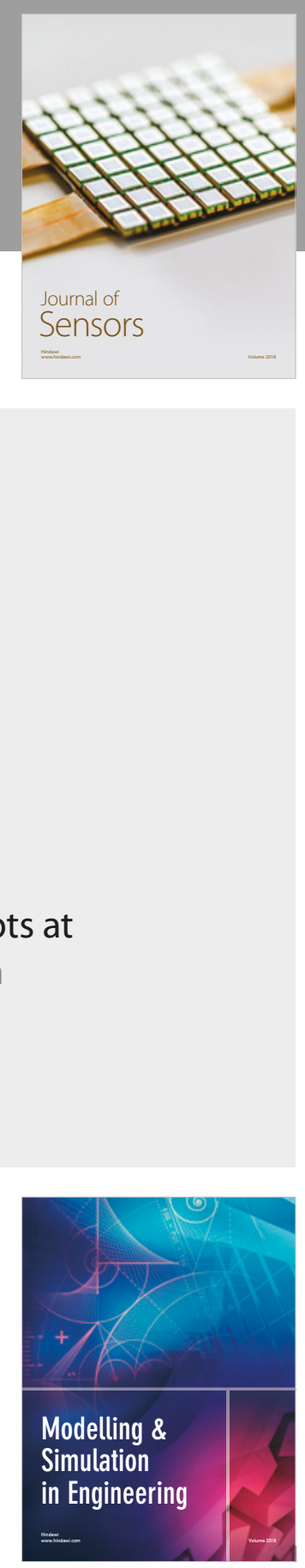

\section{Advances \\ Multimedia}
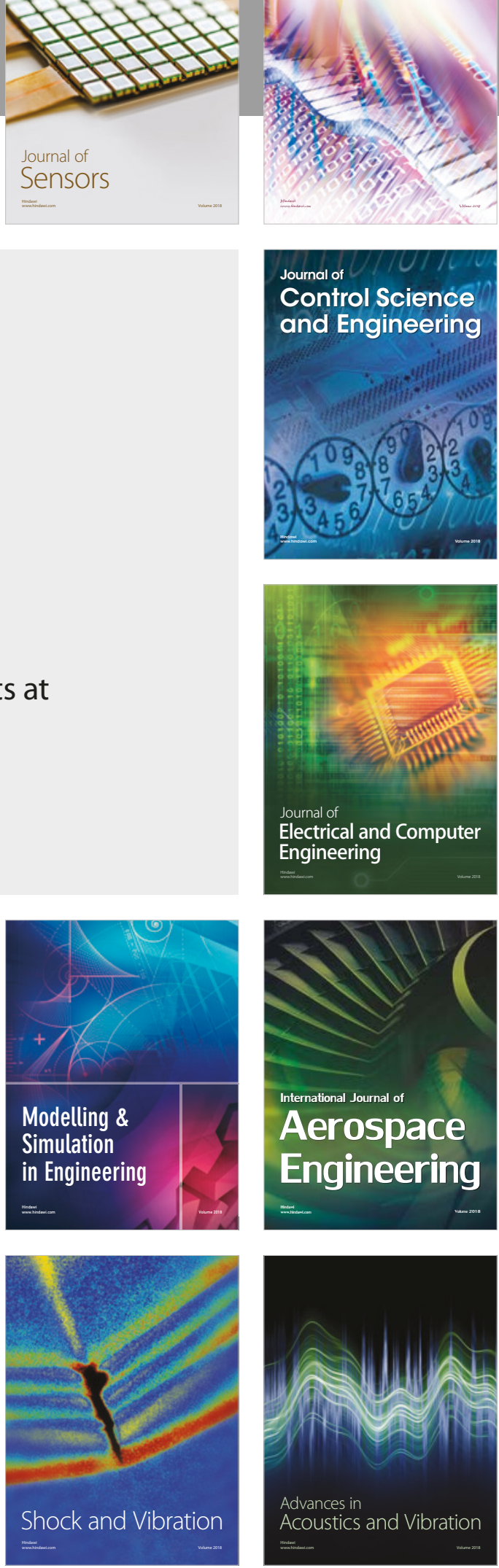